\title{
96
}

\section{Modelling Interaction for Virtual Reality Systems}

\author{
Kulwinder Kaur and Alistair Sutcliffe \\ Centre for HCI Design, City University, Northampton Square \\ London ECIV OHB \\ UNITED KINGDOM \\ K.Kaur@city.ac.uk \\ A.G.Sutcliffe@city.ac.uk
}

\begin{abstract}
There is practically no design advice on how to construct usable virtual reality systems, even though severe usability problems have been reported. This paper describes an interaction model for virtual environments developed from Norman's (1988) theory of action, as a first step in creating design guidance. The model consists of cycles of action for goal oriented, exploratory and reactive behaviour. User actions are linked to design properties required for successful interaction. The design properties represent desirable affordances of the virtual environment and can be presented as $\mathrm{HCI}$ design guidelines and in checklist questionnaires for evaluation.
\end{abstract}

KEYWORDS virtual reality, interaction models, interaction design

\section{Introduction}

Virtual Environments (VEs) are a novel interface type offering new possibilities for human-computer interaction. Notable features of VEs include their structure as 3D graphical models which the user navigates through and interacts with, typically using natural interaction styles, such as viewpoint control modelled on human head movements. However, guidance on how to design usable VEs are hard to find, even though significant usability problems have been reported. For example, in an evaluation of a submarine VE (Kaur et al. 1996) usability problems included getting and maintaining a suitable viewing angle, losing whereabouts after getting too close to objects (nose against the wall) and recognising interactive hot-spots in the environment.

\section{Modelling interaction}

As a first step in creating interface design guidance for VEs, we have developed models of interaction by elaborating Norman's (1988) generalised model of action to describe interactive behaviour in VEs.

Tasks in VEs are often loosely structured with exploration and opportunistic actions (Hayes-Roth and Hayes-Roth 1979). For example, in many simulation and tutorial applications, the user's task is to explore the environment so behaviour is primarily opportunistic following of cues. VEs are often active, with objects operating independently of the user's actions through system initiative (Bryson, 1995). Therefore, 3 inter-connected cycles have been used to model interaction:

Task/action cycle - describes purposeful, top-down behaviour in planning and carrying out specific actions as part of the user's task or goal.

Explore/navigate cycle - describes opportunistic and less goal directed behaviour when the user explores or searches for features of interest.

System initiative cycle - describes reactive behaviour to system events, and to the system taking interaction control from the user (e.g. taking the user on a pre-set tour of the environment).

Figure 1 shows a section of the explore/navigate cycle. The user scans the environment with either an intention to explore or an intention to search for an object to carry out actions from the task/action cycle. The user then continually navigates and scans until target objects are found or features in the environment arouse interest, possibly prompting exploratory actions. 


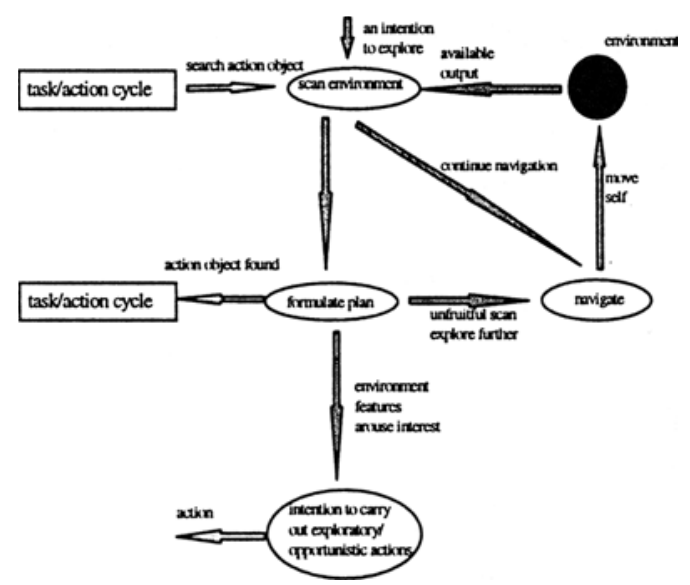

Figure 1: Section of explore/navigate cycle

The interaction models provide a clear breakdown of interactive behaviour and have been used to systematically reason about what properties are required in a design to support the user during each identified stage in interaction. Required design properties have been linked to usability problems by identifying difficulties likely to occur if the properties are missing or inadequate. For example, for the scan stage in the explore/navigate cycle, the properties 'distinguishable objects' and 'identifiable objects' are required. If these properties are not satisfied, a predicted problem is that the user will have difficulty determining what objects are in their immediate vicinity when scanning the VE.

We are evaluating the predictive power of the interaction models with experimental studies, using think aloud protocols and analysis of video footage. Observed patterns of mental and physical activity can then be checked against predictions in the interaction models. We are evaluating the importance of the design properties, by implementing them in an existing VE and then testing for differences in numbers of usability problems and task performance for users of the altered version over an original version. Results from the studies will be used to refine the interaction models and set of design properties.

\section{Discussion}

Our future work involves adapting the design properties into guidelines for design and checklist questions in evaluation methods. For example, for the scan stage in the explore/navigate cycle, a walkthrough evaluation method would include the check:

When scanning the VE, can the users distinguish and recognise many/few/none of the objects? Does the object appearance match the users' expectations?

In conclusion, an interaction modelling approach has been proposed as one promising way of addressing problems of interaction design for VEs. The models lead to design properties which are required for supporting important stages in interaction. The properties are being used to form the basis of design advice and criteria for the evaluation of VEs, from a usability perspective.

\section{ACKNOWLEDGMENTS}

Kulwinder Kaur is funded by the EPSRC.

\section{References}

Bryson S. (1995). Approaches to the successful design and implementation of VR applications, in Virtual Reality Applications, Ed Earnshaw R.A., Vince J.A. and Jones H. London, Academic Press.

Hayes-Roth B. and Hayes-Roth F. (1979). A cognitive model of planning. Cognitive Science 3, 275-310. Kaur K., Maiden N. and Sutcliffe A. (1996). Design practice and usability problems with virtual environments, in: Virtual Reality World '96 conference, Stuttgart. Proceedings. IDG Conferences. Norman D.A. (1988). The psychology of everyday things. New York, Basic Books Inc. 